\title{
Clinical Approaches and Outcomes of Serous Endometrial Cancer in a Large Tertiary Referral Centre
}

Wilkinson M, ${ }^{1}$ Corry E, ${ }^{2}$ Murphy $\mathrm{S},{ }^{1}$ Walsh T, ${ }^{1} \mathrm{M}^{\mathrm{c}}$ Vey $\mathrm{R},{ }^{1}$ Boyd $\mathrm{B},{ }^{1}$ Hewitt $\mathrm{M},{ }^{2}$ Brennan D,

(1): Mater Misericordiae University Hospital, Eccles Street, Dublin 7, Ireland. (2): Cork University Maternity Hospital, Wilton, Cork, Ireland

\section{Background}

Traditionally Serous endometrial cancer has been classified as a type 2 endometrial cancer and are typically associated with poorer outcomes. Currently there is a lack of consensus on the uniform management of Serous Endometrial cancer, owing to the fact that often these patients are poor surgical and oncology candidates. We sought to perform a retrospective analysis of the clinical approach to such tumours and their outcomes in a large tertiary referral setting.

\section{Methods}

A retrospective study from January 2009 to January 2018 of all cases of predominantly serous histology. Data parameters including treatment regimens and survival patterns were recorded.

Treatment Choices in High Grade Serous Endometrial Cancer

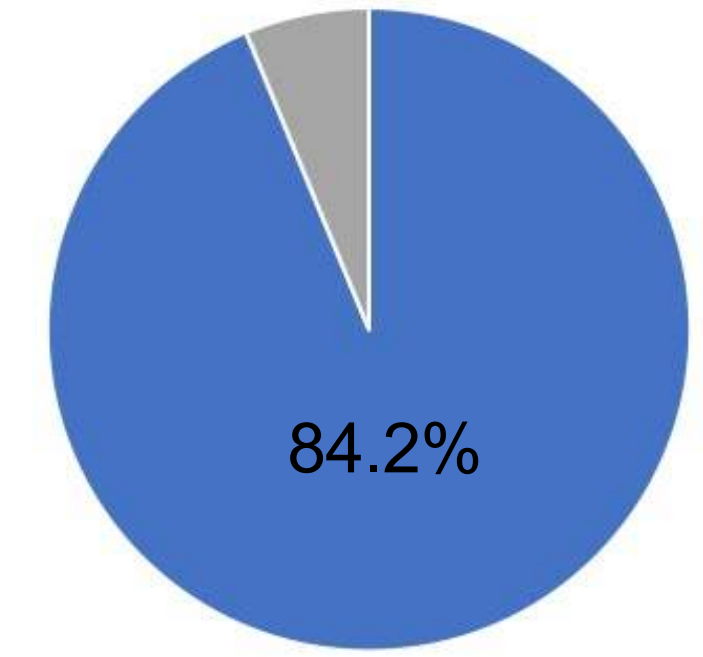

- Surgical debulking +/- Adjuvant therap

m Neoadjuvant/Palliative treatment

Figure1: $84.2 \%$ were suitable for upfront de-bulking

\section{Results}

A total of 57 patients with an average age at presentation of 67.5 years (47-92) were included in the study. Upon diagnosis, 9/57 (16\%) had an $A S A \geq 3$ and were deemed un-suitable for surgical intervention. 5/9 $(55.5 \%)$ received neoadjuvant chemotherapy, $2 / 9(22.2 \%)$ received palliative RT while 2/9 $(22.2 \%)$ declined treatment. $48 / 57$ (84.2\%) had upfront de-bulking surgery with a wide variation in the choice of adjuvant therapy; Chemo+RT 18/48 (37.5\%), Chemo Only 16/48 (33.3\%), RT only $3 / 48$ (6.2\%), Progesterone only 2/48 (4.1\%), No Therapy $9 / 48$ $(5.9 \%)$. (Figure 2 \& Table 1$)$ The 18 month progression free survival was $51.1 \%$. There was a statistically significant difference in the 18 month progression free survival between the Chemo+RT and the Chemo only group; (71\% V 42\%: $\mathrm{P}<0.05)$.

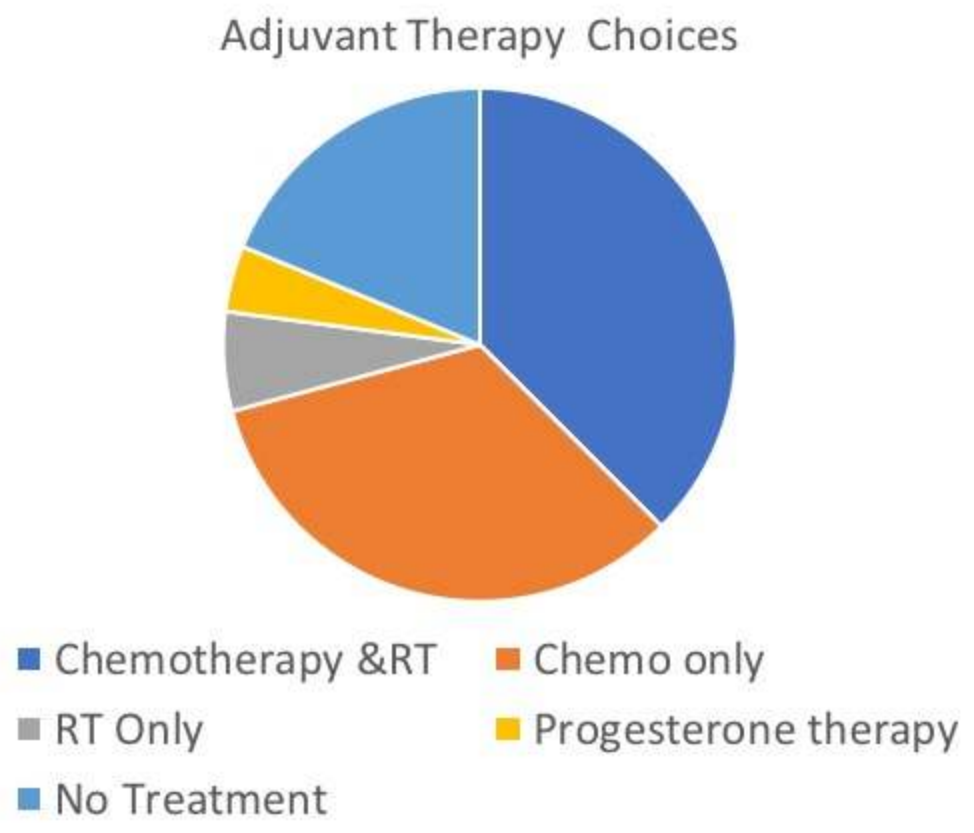

Figure 2: Combined chemotherapy and radiotherapy (37.5\%) was the most common adjuvant therapy post surgical de-bulking.

\begin{tabular}{|c|c|c|}
\hline $\begin{array}{c}\text { Adjuvant Therapy } \\
\text { Chemotherapy and RT }\end{array}$ & $\begin{array}{c}\text { Number of patients } \\
\text { (n=48) }\end{array}$ & $\%$ \\
\hline Chemotherapy Only & 18 & 37.5 \\
\hline Radiotherapy only & 3 & 33.3 \\
\hline Progesterone Therapy & 2 & 6.2 \\
\hline No Treatment & 9 & 4.1 \\
\hline
\end{tabular}

Table 1: Breakdown of treatment choices post surgical debulking.

\section{Conclusion}

A larger percentage of these patients receive neoadjuvant treatment only compared to their Endometrioid counterparts. There is a wide variation in adjuvant treatments with those receiving combined chemotherapy and radiotherapy having higher progression free survivals at 18 months. na h'Ollscoile Corcaigh 